\title{
Revista \\ Brasileira \\ de Enfermagem \\ REBEn \\ Formação em enfermagem: interface entre as diretrizes curriculares e os conteúdos de atenção básica
}

PESQUISA

Nursing education: interface between the curriculum guidelines and content of primary health attention

Educación en enfermería: interfaz entre las diretrices curiculares y los contenidos de atención básica

\author{
Maria Josefina Silva', Eliane Miranda de Sousa', Cibelly Lima Freitas ${ }^{\mathbf{I}}$ \\ 'Universidade Federal do Ceará. Departamento de Enfermagem. Programa de Pós-graduação em Enfermagem. Fortaleza, CE
}

Submissão: 19/01/2010

Aprovação: 06/09/2010

\section{RESUMO}

Este trabalho objetivou analisar Projetos Políticos Pedagógicos (PPP) dos três Cursos de Enfermagem mais antigos do Ceará, demonstrando como as Diretrizes Curriculares Nacionais de Enfermagem se expressam neles, e as transformações ocorridas na Enfermagem como profissão no Brasil. Trata-se de uma pesquisa documental, na Qual se observou a influência das Diretrizes Curriculares Nacionais de Enfermagem na formação de profissionais para atuar na Atenção Básica. Do estudo concluiu-se Que os PPP analisados se preocupam com a formação voltada às Diretrizes Curriculares Nacionais de Enfermagem, mas Que duas das Universidades em Questão possuem um currículo mais voltado aos princípios da Atenção Básica e apenas uma delas traz a proposta da integralidade curricular, aspecto Que contribui para discussões na área da educação ao considerar a formação do profissional para o Sistema Único de Saúde.

Descritores: Enfermagem; Atenção Primária à Saúde; Ensino.

ABSTRACT
This study aimed to analyze the Political and Pedagogical Projects (PPP) of three nursing courses older Ceará, showing how the National Curriculum Guidelines for Nursing are expressed in them, and the changes occurring in nursing as a profession in Brazil. This is a documentary research, in which he noted the influence of the National Curriculum Guidelines for Nursing in training professionals to work in Primary Care. From the study it was concluded that the PPP analysis are concerned with the training focused on the but two of the universities concerned have a curriculum more focused on the principles of primary care and only one of them brings the proposal of the whole curriculum, an aspect that contributes to discussions in education when considering the professional training for Single Health System.

Key words: Nursing; Primary health care; Teaching.

\section{RESUMEN}

Este estudio tuvo como objetivo analizar las políticas y proyectos pedagógicos (PPP) de tres cursos de enfermería mayores Ceará, mostrando cómo las Directrices del Currículo Nacional de Enfermería se expresan en ellos, y los cambios ocurridos en la enfermería como profesión en Brasil. Esta es una investigación documental, en la Que señaló la influencia de la Directrices del Currículo Nacional de Enfermería en la formación de profesionales para trabajar en Atención Primaria. Del estudio se concluyó Que el análisis del PPP se ocupan de la capacitación se centró en la Directrices del Currículo Nacional de Enfermería pero dos de las universidades en cuestión tienen un currículo más centrado en los principios de atención primaria y sólo uno de ellos trae la propuesta del plan de estudios conjunto, un aspecto que contribuye a los debates en la educación al considerar la formación profesional para el Sistema único de Salud.

Descriptores: Enfermería; Atención primaria de salud; Enseñanza.

AUTOR CORRESPONDENTE Cibelly Lima Freitas. Rua Marechal Humberto de Alencar Castelo Branco, 23. CEP 6204I-590. Sobral, CE. E-mail: cibellyaliny@gmail.com 


\section{INTRODUÇÃO}

No Brasil, o Movimento da Reforma Sanitária, iniciado no final da década de 70, culminou com a VIII Conferência Nacional de Saúde (CNS), realizada em Brasília, em 1986. Os temas dessa conferência foram: "Saúde para todos e dever do Estado" e "Reorganização do Sistema e Financiamento". Esta se configurou como um marco importante para a saúde no Brasil, Que passou a ser entendida como um direito do cidadão e um dever do Estado. Em 1988, foi promulgada a Nova Constituição Brasileira, dela originando-se o Sistema Único de Saúde (SUS), regulamentado pela Lei Orgânica da Saúde no .8 .080 , de 19 de setembro de $1990^{(1)}$.

O SUS é norteado pelos princípios doutrinários de universalidade, equidade e integralidade. Com isso, tornaram-se necessárias novas formas de organização dos serviços de saúde, dentre as Quais se acham a descentralização das decisões, a implementação da regionalização e hierarQuização da rede de serviços, assim como a resolutividade dos problemas de saúde que demandam a procura de atendimento no sistema formal de saúde, atitudes que exigem profissionais aptos para operacionalizá-lo ${ }^{(2)}$.

O processo de implantação do SUS trouxe modificações para a organização das práticas de atenção e de gestão do sistema de saúde, mediante a formulação e ampliação de propostas de novos modelos assistenciais, envolvendo a diversificação dos serviços de saúde, os novos processos de Qualificação dos trabalhadores e a natureza do trabalho em saúde. Assim, novos paradigmas devem nortear a formação dos trabalhadores da área ${ }^{(3)}$.

A Lei de Diretrizes e Bases da Educação Nacional, no 9.394 , de 20 de dezembro de 1996, Que estabelece as diretrizes e bases da educação nacional, configurou-se em um novo paradigma para a formação. Na Enfermagem, mais especificamente, tem-se as Diretrizes Curriculares Nacionais para a graduação em enfermagem (DCN/ENF), cujo ideal básico é a flexibilização curricular, com vistas a possibilitar uma sólida formação de acordo com o estágio do conhecimento desenvolvido em cada área, permitindo ao graduado enfrentar as rápidas mudanças na área da saúde e seus reflexos no mundo do trabalho ${ }^{(4)}$.

Nesse contexto, deve-se considerar o perfil do enfermeiro definido nas DCN/ENF como um indivíduo com formação profissional generalista, técnica, científica e humanista, com capacidade crítica e reflexiva, preparado para atuar em diferentes níveis de atenção do processo saúde-doença, pautando-se em princípios éticos ${ }^{(4)}$.

É importante considerar Que as DCN/ENF, aprovadas em 200 I, desencadearam um movimento de reestruturação dos currículos, buscando adeQuá-los às novas exigências profissionais. A reforma curricular dos cursos de graduação em enfermagem, ora ocorrendo em todo Brasil sob as referidas orientações, deixa clara a preocupação com a solidariedade e a cidadania, com o saber conviver, com o aprender a ser e o aprender a viver com o outro, elementos Que constituem a essência do humanismo e da ética como mola mestra do comportamento humano(5).

Como expressão disso, destaca-se o movimento das instituições de ensino, onde significativas mudanças também estão em curso na busca da articular ensino, serviço e movimentos organizados da população, na perspectiva de contribuir para a formação do profissional da saúde, especialmente do enfermeiro. Nesse sentido, o Projeto UNI (Nova Iniciativa na Formação dos Profissionais de
Saúde), o VER-SUS (Vivência Estágio na Realidade do Sistema Único de Saúde), o Pró-Saúde (Programa Nacional de Reorientação da Formação Profissional em Saúde), o Aprender-SUS (O SUS e os cursos de graduação na área da saúde), bem como propostas em via de implantação, como o PET-Saúde (Programa de Educação pelo Trabalho para a Saúde), são exemplos expressivos da tentativa de aproximação entre ensino, serviço e comunidade, mediante a consecução de avanços nos projetos pedagógicos articulados com práticas de saúde e princípios do SUS.

No Que diz respeito à formação profissional em saúde, o SUS assume, então, o papel de interlocutor, orientando a formulação de projetos políticos pedagógicos e não somente a função de campo de prática (estágio/aprendizagem), pressupondo a necessidade de intervenção estatal via articulação ministerial nas áreas da saúde, educação, trabalho, seguridade, meio ambiente, dentre outras, para o desenvolvimento de recursos humanos do setor ${ }^{(3)}$.

Assim, defende-se Que os trabalhadores de saúde sejam sujeitos importantes do processo histórico de implementação de novos paradigmas no Sistema Único de Saúde brasileiro e reconhece-se o importante papel Que os profissionais de enfermagem tiveram e têm na construção do SUS. Ao enfermeiro compete novas atribuições e competências dentro desse sistema, onde o profissional de enfermagem é um dos grandes alicerces para implementação das políticas em saúde. Desde já, está a importância de uma formação de Qualidade em sintonia com as Diretrizes Curriculares Nacionais e as políticas públicas de atenção à saúde, Que proporcionem uma perspectiva de inserção das competências e habilidades previstas para os profissionais da enfermagem.

Nesse contexto, é imprescindível a necessidade de acompanhar como as diretrizes em Questão vêm sendo compreendidas e implementadas na proposta pedagógica das instituições Que formam os enfermeiros, a fim de garantir a assistência humanizada à saúde, pretendida pelo SUS no atual momento.

No momento em Que o SUS completa 20 anos de implementação, busca-se saber em Que medida os trabalhadores da saúde encontram-se capacitados para exercerem atividades nesse sistema. Em outras palavras, deseja-se conhecer Que conteúdos na área da Atenção Básica são estudados nos cursos de graduação em saúde, tendo como referencial norteador as Diretrizes Curriculares Nacionais para os Cursos de Graduação em Enfermagem. Emerge daí a relevância deste trabalho Que intenta responder tais Questionamentos apoiando-se na análise dos atuais Projetos Pedagógicos de três dos mais antigos cursos de graduação em enfermagem do Estado do Ceará.

Com o estudo, espera-se suscitar reflexões Quanto ao compromisso assumido por instituições de ensino superior Quanto à formação de trabalhadores capazes de responder adequadamente à complexidade dos problemas de saúde encontrados na realidade dos serviços e de cada região, considerando um perfil Quantitativo e Qualitativo de profissionais para a consolidação do SUS.

\section{MÉTODO}

Para análise dos PPPs optou-se por desenvolver uma pesquisa documental, na Qual se utiliza materiais Que não receberam tratamento analítico, como os documentos conservados em órgãos públicos e instituições privadas. 
A coleta de dados se deu no período de janeiro a maio de 2009, por meio da análise das matrizes curriculares dos cursos superiores de formação em Enfermagem do Ceará. Salienta-se que foi solicitada autorização prévia às Coordenações de cada curso para proceder à análise dos referidos documentos, por meio de Termos de Consentimentos.

Atitude igualmente ética corresponde à atribuição de nomes fictícios às Instituições de Ensino Superior, no intuito de preservarlhes o anonimato. Destarte, deu-se-lhes os nomes Alfa, Beta e Gama para identificar os cursos estudados.

Para o processo de análise do material coletado foi utilizada a análise temática, Que estabelece três passos básicos para a investigação: a ordenação dos dados, Que engloba o conteúdo das observações realizadas, bem como o levantamento documental e bibliográfico referente ao tema; a classificação dos dados, Que é obtida através do Questionamento teórico dirigido ao campo de investigação com base numa fundamentação teórica, realizada através de leituras exaustivas e repetidas dos materiais selecionados, buscando estabelecer uma relação com do ponto de vista dialético; e a análise final, Que transita entre o concreto e o abstrato, o particular e o geral das informações recolhidas no campo de pesQuisa, voltando-se à especificidade obtida na descrição dos cursos através da análise documental ${ }^{(7)}$.

\section{RESULTADOS E DISCUSSÃO}

A análise dos Projetos Pedagógicos dos cursos permitiu realizar um breve histórico da cada curso de enfermagem; identificar a consonância entre este e o perfil do egresso, as competências e habilidades gerais e específicas, bem como as áreas do conhecimento contempladas. Identificou-se ainda a interface entre os conteúdos presentes nos referidos PPP dos cursos e a área da Atenção Básica.

\section{Contando as histórias dos cursos de enfermagem}

\section{A história do Curso de Enfermagem da Universidade Alfa}

Origina-se em Cursos de Emergência de Voluntários Socorristas e de Defesa Passiva Anti-Aérea, realizados de outubro de 1942 a 1943. Os referidos cursos se destinavam às esposas de oficiais do Exército, Marinha e Aeronáutica.

Depois de inúmeras discussões em torno da necessidade de formação de enfermeiras no Estado do Ceará, ocorreu a idéia da criação da Escola Associação São Vicente de Paulo, associação essa Que se tornaria a entidade mantenedora da instituição. Então, a nova escola iniciou seu curso regular, tornando-se a primeira escola de enfermagem no Nordeste. Em 1975, essa escola encampada pelo Governo do Estado com toda sua Estrutura e Organização, passou a denominar-se Curso de Enfermagem da Universidade Alfa, permanecendo fundamentada no currículo vigente na época, no Qual predominava o modelo clínico de assistência médica individual, curativa e hospitalar, enfocando os aspectos biológicos, em detrimento do ensino das Ciências Humanas e Sociais.

O Curso de Enfermagem da Universidade Alfa não passou por nenhuma reforma no período de 1982 a 1984. Em 1988, não foram promovidas alterações estruturais em sua matriz curricular, apenas introduziram-se disciplinas referentes à Licenciatura Que, à época, era realizado concomitantemente com o bacharelado. No primeiro semestre de 1997, para atender a uma resolução do MEC, foi iniciada a revisão do currículo vigente e a elaboração de um PPP.

O PPP do Curso de Enfermagem desta Universidade foi elaborado tomando por base a Lei de Diretrizes e Bases Curriculares Nacionais dos Cursos de Graduação em Enfermagem (Parecer CNE/ CES 1.133/2001). Isso culminou em seu desenho curricular atual organizado por disciplinas, sendo $4.230 \mathrm{~h}$ obrigatórias e $120 \mathrm{~h}$ optativas, distribuídas em nove semestres. Este desenho participa do Programa de Reorientação para a Formação em Enfermagem PRÓ-SAÚDE II, o Que revela uma preocupação com a formação do enfermeiro.

\section{A História do Curso de Enfermagem da Universidade Beta}

O curso nasce, na década de 1970, na esteira da meta de extensão de cobertura da Organização Mundial de Saúde para países do então terceiro mundo, sendo a Enfermagem colocada como estratégica para tal fim, de acordo com pronunciamentos de organismos internacionais. O funcionamento do curso baseia-se no Documento sobre Desenvolvimento do Ensino Superior de Enfermagem no Brasil, do MEC.

O funcionamento do referido curso iniciou-se em $1^{\circ}$ de março de 1976, com o processo formativo de sua primeira turma. Em dezembro de 1978, o currículo pleno do curso é aprovado, sendo encaminhado para o Conselho Federal de Educação, sob forma do processo $\mathrm{n}^{\circ} 7.727 / 78$, de 8 de dezembro de 1978. O reconhecimento do curso pelo MEC se dá através da Portaria no 1069 , de 29 de outubro de 1979, publicada no Diário da União de 30/10/ 79.

$\mathrm{Na}$ atualidade, os conteúdos do currículo estão organizados de forma integralizada, perfazendo uma carga horária total de 4.320h, sendo 3.963 de disciplinas teóricas e 384 horas de atividades complementares, distribuídas em nove semestres e guardando uma especificidade de organização por ciclos de vida. Esta universidade participa do PRÓ-SAÚDE I e II, e encontra-se em processo de reforma curricular.

\section{A História do Curso de Enfermagem da Universidade Gama}

O Curso de Enfermagem desta IES foi fundado no ano de 1973 , reconhecido posteriormente pelo Decreto-lei no 79.018/76. Achava-se vinculado ao Centro de Ciências da Saúde com uma oferta inicial de 30 vagas. Trata-se do segundo curso a ser criado no estado do Ceará.

Desde o ano de sua instalação, em 1973, o Curso de Enfermagem tem passado por efetivas alterações curriculares, incluindo novas disciplinas e modificando a carga horária de algumas. Em 1995, foi implantado o novo currículo, conforme Parecer CFE no 3 1 4/94 e Portaria Ministerial no 172 1/94, visando adequar o ensino às demandas sociais e políticas. A organização do atual currículo tem como base a Resolução CNE/CES No 3, de 07 de novembro de 2001, Que estabelece um currículo de enfermagem formado por 3 (três) grandes áreas temáticas: Ciências Biológicas e da Saúde, Ciências Humanas e Sociais, Ciências da Enfermagem.

\section{Perfil dos egressos dos cursos de enfermagem}

Foi analisado nos PPP dos Cursos o texto Que define o perfil dos egressos. Para fins de análise, criou-se categorias analíticas 
denominadas: "Contempla Totalmente", "Contempla Parcialmente" e "Não Contempla", para dizer em Que medida estão contemplando os aspectos destacados nas Diretrizes Curriculares Nacionais do Curso de Graduação em Enfermagem, Que diz:

Art. $3^{\circ} \mathrm{O}$ Curso de Graduação em Enfermagem tem como perfil do formando egresso/profissional:

I - Enfermeiro, com formação generalista, humanista, crítica e reflexiva. Profissional Qualificado para o exercício de Enfermagem, com base no rigor científico e intelectual e pautado em princípios éticos. Capaz de conhecer e intervir sobre os problemas/situações de saúde-doença mais prevalentes no perfil epidemiológico nacional, com ênfase na sua região de atuação, identificando as dimensões bio-psico-sociais dos seus determinantes. Capacitado a atuar, com senso de responsabilidade social e compromisso com a cidadania, como promotor da saúde integral do ser humano; $e$

II - Enfermeiro com Licenciatura em Enfermagem capacitado para atuar na Educação Básica e na Educação Profissional em Enfermagem.

Tendo como base a definição do perfil do egresso, viu-se Que todos os PPPs das três Universidades em estudo a contemplam. Ao referir-se ao perfil do enfermeiro com formação generalista, humanista, crítica, reflexiva, o Artigo $3^{\circ}$ deixa a impressão de aludir a uma pessoa aberta, com uma objetividade reflexiva voltada para o sentido humano, uma pessoa para o mundo ${ }^{(3)}$.

$\mathrm{Na}$ análise de cada trecho referente ao perfil dos egressos, podese constatar que a Universidade Gama fez uma abordagem que "Contemplou Totalmente" as DCN/ENF em relação ao perfil do egresso. Abordou em tópicos, mais detalhadamente, elementos como a Questão ética, a formação generalista, crítica e reflexiva; o processo saúde-doença, a educação permanente, a promoção, proteção e recuperação da saúde. Já as Universidades Beta e Gama "Contemplaram parcialmente", uma vez Que, em seus respectivos Projetos está posta apenas uma breve descrição sobre o perfil do egresso.

O profissional generalista é aQuele Que tem uma visão mais global e menos específica da Enfermagem, incorporando saberes epidemiológicos, de educação em saúde, de trabalho em grupo e de gestão, de forma a ajudá-lo nas intervenções individual e coleti$\mathrm{va}^{(8)}$. Na formação humanista, a enfermagem deve congregar a pesQuisa, o ensino, a extensão e a assistência, resgatando o respeito pela vida, em especial, humana.

A formação crítica do profissional o estimula a desenvolver atividades com objetividade, preparando-o para decisões competentes, baseadas em atitudes Questionadoras. Já a formação reflexiva pode se apresentar por meio da postura ética, não considerando só a normatização de deveres e direitos ou regras de comportamento profissional.

O egresso do curso de enfermagem precisa ser promotor da saúde integral do ser humano tendo sempre como base o rigor científico; sua atuação deve estar pautada em princípios éticos e os campos de práticas e estágios se mostram ricos em situações concretas. Além da necessidade de ser conhecedor e interventor do perfil epidemiológico nacional/regional e local onde a "diversificação de cenários de prática, ampliação dos tempos de prática e aproximação ao SUS, além da orientação do perfil ético e humanístico dos profissionais e à multiprofissionalidade, em especial com o caráter interdisciplinar, direcionarão o futuro enfermeiro a não só conhecer o perfil epidemiológico, mas tornar-se um interventor desse perfil, contribuindo para a melhoria da eualidade de vida dos cidadãos" ${ }^{(8)}$.

Competências e habilidades gerais e específicas do enfermeiro

As diretrizes curriculares podem ser definidas como diretrizes gerais Que orientam o planejamento acadêmico do curso de graduação para formação profissional. No campo da enfermagem, as DCN preconizam ao profissional uma formação generalista, crítica e reflexiva, capaz de possibilitar-lhe conhecer e intervir sobre os problemas/situações de saúde-doença mais prevalentes no perfil epidemiológico nacional, com ênfase na sua região de atuação, identificando as dimensões biopsicossociais de suas determinantes ${ }^{(9)}$.

Competência é a capacidade para aplicar adequadamente conhecimentos e habilidades para alcançar um determinado resultado em um contexto concreto. Desenvolver competências para o pleno exercício de um ofício requer interagir no âmbito ou contexto em Que se realiza esse ofício, aferindo a progressiva Qualificação do aprendiz para o desempenho desse mesmo ofício ${ }^{(10)}$.

Há dois fortes obstáculos à implementação das diretrizes curriculares na forma almejada pela lei: a dicotomia entre teoria e prática e a dificuldade de avaliação de competências profissionais para efeito de certificação educacional. A efetiva integração entre os processos de ensino-aprendizagem e de produção de serviços é, ademais, requisito indispensável para o desenvolvimento de competências profissionais, meio de cultura insubstituível para germinação de práticas adeQuadas de avaliação dessas mesmas competências $^{(10)}$.

$\mathrm{Na}$ análise dos PPPs das Universidades Alfa, Beta e Gama, foram Quantificadas as competências e habilidades gerais e específicas listadas nos documentos oficiais. Além disso, foi analisada a sua similaridade aos princípios contemplados nas Diretrizes Curriculares Nacionais (Quadro I).

Na leitura do Quantitativo de competências gerais pontuadas pelos PPPs em foco, apenas a Universidade Gama pontuou as competências e habilidades gerais conforme o artigo $4^{\circ}$ da resolução CNE/CES No3, de 7 de novembro de 2001. As universidades Alfa e Beta não contemplaram nenhuma das competências e habilidades gerais.

No Que se refere à análise das competências e habilidades específicas, notou-se que na Universidade Alfa foram pontuadas 15 competências e habilidades específicas, então "Contemplou parcialmente"; enquanto a Universidade Beta "Contemplou totalmente", indicando em seu Projeto 60 competências e habilidades específicas.

A Universidade Gama "Não Contemplou" nenhuma das competências e habilidades específicas, ou seja, não está pontuado Quais seriam as competências e habilidades específicas para o enfermeiro Que se forma nessa Universidade.

Apesar de, durante a análise dos documentos, muitas dessas competências estarem subentendidas em alguns trechos dos textos, não houve nenhuma Universidade Que "Contemplasse Totalmente" todas as competências e habilidades gerais e específicas 
Quadro 1. Competências e Habilidades Gerais e Específicas nas universidades em estudo. Fortaleza, CE, 2009.

\begin{tabular}{|l|c|c|}
\hline Universidades & $\begin{array}{c}\text { Competências e } \\
\text { habilidades gerais }\end{array}$ & $\begin{array}{c}\text { Competências e habilidades } \\
\text { espećficas }\end{array}$ \\
\hline Alfa & Não Contempla & Contempla Parcialmente \\
\hline Beta & Não Contempla & Contempla Totalmente \\
\hline Gama & Contempla Totalmente & Não Contempla \\
\hline
\end{tabular}

em seus Projetos Pedagógicos, ou seja, nenhuma delas resgatou os aspectos tal como estão descritos nas DCN/ENF.

As Diretrizes Curriculares Nacionais do Curso de Graduação em Enfermagem trazem, entre outros, três grandes artigos referentes às competências e habilidades gerais, específicas e conteúdos essenciais para a formação do enfermeiro (artigos $4^{\circ}, 5^{\circ}$ e $6^{\circ}$, respectivamente) onde se percebe um delineamento centrado no humanismo-existencialista ${ }^{(5)}$.

Em relação às competências e habilidades gerais requeridas para o exercício profissional, o Artigo $4^{\circ}$ faz referência, embora Que de forma implícita nos Quatro primeiros incisos, ao aprendizado Que valorize Questões ético-humanistas: atenção à saúde (prática integrada e contínua no nível individual e coletivo); tomada de decisões (uso apropriado, eficácia e custo-efetividade dos materiais, instrumentos e da força de trabalho); comunicação (interação com outros profissionais e com o público geral, além do domínio de tecnologias de comunicação e informação); liderança (responsabilidade, empatia e habilidade para tomar decisões). As competências de ordem técnica e Que demandam a apreensão das anteriores são abordadas mais especificamente nos incisos $\mathrm{V}$ e VI, relacionados, respectivamente, com a administração e gerenciamento (aptidão para o gerenciamento e administração da força de trabalho e dos recursos físicos e materiais); educação permanente (capacidade de aprender continuamente e de formar redes de cooperação) dos profissionais ${ }^{(5)}$.

Essas orientações gerais se configuram como propostas de referência para uma formação profissional abrangente, embasada por uma concepção ampla de saúde, Que enfatiza a integralidade pela superação da dicotomia entre a abordagem epidemiológica e a clínica, o estabelecimento de boas práticas relacionais entre os sujeitos (profissionais e usuários), o uso adequado e eficiente dos recursos, o interesse em atualização contínua e a responsabilidade com a educação dos demais profissionais de saúde. Correspondem a um leque de exigências e padrões definidos como elementos indispensáveis ao perfil de um novo profissional, capacitado e responsável por uma atenção à saúde mais justa, igualitária e de melhor Qualidade para todos ${ }^{(3)}$.

\section{Áreas de conhecimento contempladas nos currículos e sua interface com a área da Atenção Básica}

Os PPPs em Questão têm em comum a forma de organização curricular, todos contemplam os conteúdos distribuídos em disciplinas. O currículo integrado está expresso no Curso de Enfermagem da Universidade Beta, contemplando uma estrutura Que atende às Diretrizes Nacionais de Enfermagem, relacionando o pedagógi- co e o corpo científico da enfermagem.

Defini-se currículo integrado como um plano pedagógico e sua correspondente organização institucional Que articula dinamicamente trabalho e ensino, prática e teoria, ensino e comunidade. As relações entre trabalho e ensino, entre os problemas e suas hipóteses de solução devem ter sempre, como pano de fundo, as características sócio-culturais do meio em que este processo se desenvolve. A proposta de currículo integrado parece mais apropriada para atender à necessidade de integrar ensino e trabalho na formação de pessoal de níveis médio e elementar, pelas instituições de saúde nas suas diversas categorias ${ }^{(1)}$.

Conforme o artigo $6^{\circ}$ da Resolução CNE/CES N³ (2001) "os conteúdos essenciais para o currículo de graduação em Enfermagem devem estar relacionados com todo o processo de saúdedoença do cidadão, da família e da comunidade..." devendo contemplar:

I - Ciências Biológicas e da Saúde - inclui os conteúdos (teóricos e práticos) de bases moleculares e celulares dos processos normais e alterados da estrutura e da função do corpo humano, aplicados às situações decorrentes do processo de saúde-doença. II- Ciências Humanas e Sociais - inclui os conteúdos necessários à compreensão das relações do homem com a sociedade $e$ os determinantes sociais, culturais, econômicos, ético-legais, políticos do processo de saúde-doença.

III-Ciências de Enfermagem:

I - Fundamentos de Enfermagem - conteúdos técnicos e metodológicos e instrumentos inerentes ao trabalho do enfermeiro a nível individual e coletivo.

2- Assistência de Enfermagem - conteúdos teóricos e práticos necessários para a assistência de Enfermagem à criança, ao adulto, ao adolescente, à mulher e ao idoso, estabelecendo intervenções de cuidado de enfermagem. Levando em consideração os aspectos bio-psico-sociais e o processo saúde-doença.

3- Administração de Enfermagem - envolve os conteúdos teóricos e práticos da administração de enfermagem e gerenciamento desenvolvidos na rede de saúde.

4- Ensino de Enfermagem - os conteúdos referentes à instrumentalização do enfermeiro para exercer a prática educativa.

A análise dos PPPs permitiu perceber Que as disciplinas dos cursos em foco estão "divididas", considerando o Ciclo Básico, Que compreende ao período inicial do curso, com duração média de dois anos ou Quatro semestres e o Ciclo Profissionalizante, Que introduz as disciplinas mais específicas da Enfermagem com atuação nos campos de prática.

Viu-se Que um dos problemas detectados nos currículos era sua fragmentação em ciclo básico e ciclo profissionalizante, uma vez Que seus estudos sobre saberes e práticas curriculares em saúde, a divisão entre ambos os ciclos é um resQuício da reforma do Ensino Superior de 1968, Que instituiu os departamentos e criou a divisão dos ciclos para uma maior racionalização de recursos ${ }^{(12)}$.

Essa análise foi feita a partir das ementas de cada disciplina correspondente, fazendo uma interface com os pressupostos da Atenção Básica à Saúde proposta pelo SUS, contemplando con- 
teúdos referentes à promoção da saúde, à prevenção de doenças e agravos, à assistência e ao tratamento e à reabilitação da saúde ${ }^{(2)}$.

A participação do enfermeiro na Atenção Básica, por tratar-se de um profissional historicamente marcado pelo compromisso com a saúde pública, tem grande capilaridade e está presente na maioria das ações, especialmente no atual contexto de transformações constantes, onde é o profissional mais requisitado para assumir papeis estratégicos na implantação das políticas de saúde ${ }^{(13)}$.

Numa abordagem psico-pedagógica, o significado de currículo passa pela proposta de um conteúdo lógico, seqüencial, contínuo, pautado por objetivos educacionais previamente definidos, vivenciados através das experiências de aprendizagem organizadas pelo professor. Esta modalidade de currículo se identifica com uma visão conservadora onde prevalece o enfoque instrucional e metodológico. $\mathrm{Na}$ perspectiva deste enfoque tecnicista, o currículo tem como pressuposto a previsão, a pré-determinação e o planejamento, buscando atingir requisitos científicos de rigor, exatidão objetividade $^{(14)}$.

Mais recentemente a teoria curricular e a educacional apontam a necessidade de se compreender o currículo além de suas dimensões técnico-pedagógicas, entendendo-o como um ato de renovação e compromisso social. Esta modalidade incorpora a noção de um currículo-formação voltada para a consciência crítica, emancipação e humanização do homem, assumindo Questões de natureza ética, política, social e não apenas as de ordem técnicainstrumental. Esta linha de orientação teórica se opõe ao fato de considerar o currículo como um ato isolado de um compromisso da escola para com seus alunos e a comunidade; coloca a possibilidade de entendê-lo como um ato social, um compromisso para com uma determinada comunidade ocupacional, conduzido por diferentes postulados, prática e valores sociais Que irão mediar a formação e desempenho profissional ${ }^{(14)}$.

A partir desses eixos as ementas foram analisadas. Cada ementa foi lida e criteriosamente sistematizada nas categorias do Quadro 2.

A Universidade Alfa totalizou dezessete disciplinas nas Quais os aspectos da Atenção Básica estavam sendo citados e/ou contemplados. Destas, um total de oito disciplinas foram referentes ao período inicial do curso e nove no período profissionalizante. A Universidade Beta totalizou vinte e uma disciplinas, onde doze foram relacionadas ao ciclo básico e nove foram do ciclo profissionalmente.

A Universidade Gama foi a Que demonstrou o menor número

Quadro 2. Quantidade de disciplinas curriculares obrigatórias correspondentes ao Ciclo Básico e ao Ciclo Profissionalizante que fazem interface com a área da Atenção Básica à Saúde. Fortaleza, CE, 2009.

\begin{tabular}{|l|c|c|c|}
\hline Universidades & Ciclo básico & $\begin{array}{c}\text { Ciclo } \\
\text { profissionalizante }\end{array}$ & Total \\
\hline Alfa & 8 disciplinas & 9 disciplinas & 17 disciplinas \\
\hline Beta & 12 disciplinas & 9 disciplinas & 21 disciplinas \\
\hline Gama & 6 disciplinas & 8 disciplinas & 14 disciplinas \\
\hline
\end{tabular}

de disciplinas Que problematizam a Questão das novas diretrizes para formação de enfermeiros. É necessária a utilização de estratégias Que aproximem a formação do enfermeiro para as necessidades locais/regionais e nacionais em saúde.

Os recursos humanos em saúde precisam estar pautados nos princípios Que norteiam a política de Saúde do SUS para a consolidação do mesmo. As novas conformações para a formação do(a) enfermeiro(a) não se limitam às Questões técnicas, relativas a conteúdos de ensino, procedimentos didáticos e técnicas pedagógicas - pedagogia tecnicista. Elas pautam-se na adoção de referencial teórico- pedagógico Que sustente uma aprendizagem significativa, transformadora e adequada às demandas sociais e profissionais Que se apresentam na atualidade.

Essas análises mostram Que, das Universidades em estudo, Alfa e Beta foram as Que tiveram seus currículos mais estruturados dentro dos preceitos da Atenção Básica, de acordo com o Que está explícito nas ementas das disciplinas. É importante ressaltar Que essa análise é apenas o reflexo dos documentos estudados e, de maneira geral, isso reflete Quem serão os profissionais Que estarão abastecendo os recursos humanos para a saúde no Brasil.

\section{CONSIDERAÇÕES FINAIS}

Construir essa análise sobre a interface dos PPPs dos cursos de Enfermagem de Universidades cearenses com a Atenção Básica da Saúde no Brasil foi uma tarefa desafiadora. A partir dela constatou-se que os PPPs em estudo tentam fazer uma interface com a proposta das Diretrizes Curriculares Nacionais, o Que representa algum avanço na forma como docentes e discentes percebem as políticas de saúde do país.

Há, também, uma preocupação em fazer essa aproximação com a Atenção Básica da Saúde. Nas Universidades Alfa e Beta, esse dado reflete o nível de excelência dessas instituições e sua credibilidade na formação dos profissionais de enfermagem no estado do Ceará. Os resultados mostram Que a Universidade Beta está adiante nessa discussão Quando propôs uma forma de estruturação do currículo integrada.

Convém salientar a importância da construção de currículos majoritariamente integrados, sem disciplinas isoladas, obtendo conteúdos organizados em módulos, sem a precedência cronológica do ciclo básico. Os temas são abordados de modo que as áreas básicas funcionem como efetiva base e referência para a busca dos conhecimentos voltados à solução de problemas. $\mathrm{O}$ currículo deve representar a visão de mundo percebida pela escola e seus professores.

Para finalizar, embora as Políticas de Atenção Básica tenham se consolidado cada vez mais, ainda existe um grande desafio Quanto à formação dos profissionais que estarão atuando na Atenção Básica. Nesse sentido, os currículos das Universidades são o reflexo da formação dos profissionais Que estarão atuando para a consolidação do Sistema Único de Saúde-SUS no Brasil. Professores e estudantes devem estar comprometidos com as transformações Que são imprescindíveis para diminuir a dicotomia entre teoria e prática durante a formação acadêmica, entre discurso e ação concreta na realidade. É necessário o debate contínuo no interior do SUS, no 
interior da sociedade civil brasileira e no interior das universidades para Que se possa reconhecer minorar a fragilidade do processo de construção social da saúde no Brasil.

É um grande desafio continuar-se avaliando e melhorando a Qua- lidade dos currículos dos cursos superiores para Que a enfermagem seja atuante no processo de transformação do cenário da saúde no Brasil, e que a formação transcenda as Questões técnicas e acadêmicas, mas Que sirva como estratégia para a transformação social.

\section{REFERÊNCIAS}

1. Chioro A, Scaff A. A implantação do Sistema Único de Saúde. Brasília: Ministério da Saúde; 1999.

2. Ministério da Saúde (BB). Gestão Municipal de Saúde: leis, normas e portarias atuais. Brasília: Ministério da Saúde; 2001

3. Costa RKS, Miranda FAN. Formação Profissional no SUS: oportunidades de mudanças na perspectiva da estratégia de saúde da família. Trab Educ Saúde 2009; 6(3): 503-17.

4. Ministério da Educação (BR). Instituto Nacional de Estudos e Pesquisas Educacionais Anísio Teixeira. O ensino de enfermagem no Brasil: o ontem, o hoje e o amanhã. Brasília: Ministério da Educação; 2006.

5. Ribeiro IP, Tavares M, Esperidião E, Munari DB. Análise das diretrizes curriculares: uma visão humanista na formação do enfermeiro. Rev Enferm UERJ 2005; 13(3).

6. Gil AC. Como elaborar projetos de pesquisa. São Paulo: Atlas; 1996.

7. Minayo MCS. O desafio do conhecimento: pesQuisa Qualitativa em saúde. 9a ed. São Paulo: Hucitec; 2006.

8. Santos SSC. Perfil de egresso de Curso de Enfermagem nas Diretrizes Curriculares Nacionais: uma aproximação. Rev Bras

Enferm 2006; 59(2).

9. Ministério da Educação (BR). Comissão de Especialistas de Ensino de Enfermagem, CEEENF. Diretrizes Curriculares Nacionais para o curso de Enfermagem. Brasília: Ministério da Educação; 2000.

10. Santana IP, Campos FE, Sena RR. Formação profissional em saúde: desafios para a universidade [monografia]. Belo Horizonte: Universidade Federal de Minas Gerais; 2002.

I I. Davini MC. Currículo Integrado [monografia]. Belo Horizonte: Universidade Federal de Minas Gerais; 2002.

12. Lemos CLS, Fonseca SG. Saberes e práticas curriculares: um estudo de um curso superior na área da saúde. Interface 2009; I3(28).

13. Ministério da Saúde (BR). Secretaria de Atenção à Saúde. Departamento de Atenção Básica. Política Nacional de Atenção Básica. Brasília: Ministério da Saúde; 2007.

14. Vendrúscolo DMS, Manzolli MC. O currículo na e da enfermagem: por onde começar e recomeçar. Rev Latino-am Enfermagem 1996; 4(1): 55-70. 